\title{
Chinese Stock Price and Volatility Predictions with Multiple Technical Indicators
}

\author{
Qin Qin, Qing-Guo Wang, Shuzhi Sam Ge, Ganesh Ramakrishnan
}

Department of Electrical and Computer Engineering, National University of Singapore, Singapore City, Singapore. Email: elewqg@nus.edu.sg

Received July $19^{\text {th }}$, 2011; revised September $19^{\text {th }}, 2011$; accepted October $8^{\text {th }}, 2011$.

\begin{abstract}
While a large number of studies have been reported in the literature with reference to the use of Regression model and Artificial Neural Network (ANN) models in predicting stock prices in western countries, the Chinese stock market is much less studied. Note that the latter is growing rapidly, will overtake USA one in 20 - 30 years time and thus becomes a very important place for investors worldwide. In this paper, an attempt is made at predicting the Shanghai Composite Index returns and price volatility, on a daily and weekly basis. In the paper, two different types of prediction models, namely the Regression and Neural Network models are used for the prediction task and multiple technical indicators are included in the models as inputs. The performances of the two models are compared and evaluated in terms of directional accuracy. Their performances are also rigorously compared in terms of economic criteria like annualized return rate (ARR) from simulated trading. In this paper, both trading with and without short selling has been considered, and the results show in most cases, trading with short selling leads to higher profits. Also, both the cases with and without commission costs are discussed to show the effects of commission costs when the trading systems are in actual use.
\end{abstract}

Keywords: Regression Model, Artificial Neural Network Model, Chinese Stock Market, Technical Indicators, Volatility

\section{Introduction}

From the beginning of time it has been man's common goal to make his life easier. The prevailing notion in society is that wealth brings comfort and luxury, so it is not surprising that there has been so much work done on ways to predict the markets. Various technical, fundamental, and statistical indicators have been proposed and used with varying results. However, no one technique or combination of techniques has been successful enough to consistently "beat the market". As a result, there is a huge motivation to develop new forecasting techniques that can unravel the market's mysteries and obtain greater profits.

The stock market is known as the "cradle of capitalism". It is a place where companies come to raise their share capital and investors go to invest their surplus funds. Vast amounts of capital are invested and traded in everyday all over the world. The prediction of the stock market movements, however, poses a challenge to academicians and practitioners. The reason is that stock market movements are characterized as being uncertain and complex as it can be affected by virtually any economic, social or political development that has a bearing on the economy. This uncertainty and complexity is undesirable for any trader who is attempting to make profits from the stock market. Therefore, there is a need to reduce this uncertainty by making accurate predictions.

Initially, stock market research encapsulated two elemental trading techniques namely the Technical and Fundamental approaches [1]. In Technical analysis, it is believed that market timing is keypoint. It involves the study of historical data of the stock market to predict trends in price and volume. In other words, there is heavy reliance on historical data in order to predict future outcomes. Fundamental analysis, on the other hand involves making estimates on the intrinsic value of a stock. This technique uses information such as earnings, ratios, and management effectiveness to predict future outcomes.

As the level of investing and trading grew, there began a pursuit for better tools and methods that could not only increase gains but also minimize the risks undertaken by the investor. Tools that used modeling techniques to discover patterns within the historical data of the stock market were put to test, with an attempt to predict and bene- 
fit from the market's direction. One such example is the Linear Time Series Models, where univariate and multivariate regression models [2] were used to identify patterns in the historical data of the stock market. For nonlinear patterns, Machine Learning Models [3], in particular neural networks, were commonly used. For example, one sees that:

- In [4], the authors used a mean reverting characteristic to model and estimate the stock markets. The authors stated that the random walk which is used to describe the stock markets may not be correct when the process of stock markets diverge over time. The mean reverting characteristic is a good way to model and estimate the stock markets. The authors used two methods to estimate the parameters, which are Least Square Estimation and Maximum Likelihood Estimation. In this paper, the authors focused on the monthly data of Dow Jones Industrial Average and the Singapore Straits Times Index and got some interesting conclusion.

- In [5], the authors predicted the mid-term price trend for Taiwan stock market. The authors firstly extracted the features from ARIMA analyses, then the authors used the features which are produced in the first step to train a recurrent neural network. The Taiwan stock market series is regarded by the authors as a nonlinear ARIMA $(1,2,1)$. The conclusion of this paper is that the prediction system can predict the Taiwan stock market trend of up to 6 weeks based on four years weekly data with an acceptable accuracy.

- In [6], the authors focused the research work on Shanghai stock market for Chinese stock market is one of fast growing stock markets in the world. The authors used two types of models which are the model of stochastic SARIMA and the model of backpropagation network. The author used the actual data of Shanghai Composite Index to do the prediction and found that SARIMA model is more optimistic.

- In [7], the authors took advantage of the nonlinear dynamical theory to use the multivariate nonlinear prediction method. The prediction system is based on the reconstruction of multidimensional phase space. The authors set the model using multivariate nonlinear prediction method and got the experiment results using the data of Shenzhen Index. The authors compared the results obtained using multivariate nonlinear prediction method with the results obtained using unvariate nonlinear prediction method and found that the performance of multivariate nonlinear prediction method is better than the performance of unvariate nonlinear pre- diction method.

- In [8], the author stated that the stock market is a very complicated nonlinear system, the artificial neural network also has nonlinear characteristic. It is proper to use artificial neural network to do the prediction of stock market. The authors used the artificial neural network to imitate the trading process of stock market. Because the convergent speed of backpropagation algorithm is low, the authors enhanced the convergent speed of backpropagation algorithm by proposing the rate of deviation. The authors used the data of both Shanghai and Shenzhen to do the prediction.

- In [9], the authors explored a new method to estimate the systematic risk (which is called as beta) in China stock market. A technique is involved in this new method, which is maximal overlap discrete wavelet transform (MODWT). The technique will not lose any information when it is investigating the behavior of beta at different time frames. The experimental results showed that China stock market is quite different from other stock markets. The authors drew a conclusion that the difference between China stock market and other stock markets is due to the character and behavior finance.

- In [10], the authors analyzed the volatility of a stock in China on its returns series using the models of GARCH family. They found that the series of stock returns is stationary, and it has a significant ARCH effect, a volatility cluster exists in China stock market. The authors also found that a return of negative shock produces more volatility than the positive one of equal magnitude. They finally drew a conclusion that there is a leverage effect in stock returns volatility.

- In [11], the authors used the daily data of Shanghai stocks to do the prediction based on the family GARCH models. The paper used ME, MAE and RMSE for error measurement. From the results, the authors found that in the training period, EGAR$\mathrm{CH}-\mathrm{M}$ model can generate best performance, while in testing period, simple GARCH model or asymmetric model can produce best performance.

In general, most of stock market studies in the literature have been focused on developed markets while emerging markets are much less studied. Note that the latter is growing rapidly, and in particular, China market will overtake USA one in 20 - 30 years time and it has becomes a very important place for investors worldwide. It is thus timely to study this market's performance and efficiency based on recent data. This paper attempts to predict the Shanghai Composite Index return and volatility on a daily and weekly basis with use of multiple technical 
indicators. Specifically, the present work contributes to the literature in the following ways:

1) An attempt is made to understand the efficacy of an emerging market such as China. Today, China is one of the fastest growing emerging economies in the world. Not only is there a significant growth in the demand for investment funds but the growth in capital markets is also expected to play an increasingly important role in the process. At this transitional stage, it is necessary to assess the level of efficiency of the Chinese Stock Market in order to establish its longer term role in the process of economic development. However as studies on Chinese Stock Markets are very few and also dated and mostly inconclusive, the objective of this study in this paper is to test whether predictability of return rates and price volatility is possible.

2) An attempt is made to predict stock market price volatility. Volatility is an important indicator for investors. Results from this study do show that neural network models have their merits and perform better than regression models.

3) Multiple technical indicators are used in modeling. We also use different combinations of different technical indicators to do the prediction to see the performance. Some combinations improve the performance of the prediction.

The rest of the paper is organized as follows. Section 2 gives an overview of the stock market prediction methods. Section 3 presents the methodology and shows the results for the predictability of Shanghai Composite Index return. Section 4 presents the methodology and shows the results for the predictability of Shanghai Composite Index price volatility. Finally, Section 5 gives a conclusion of the work that has been done, as well as possible areas of improvements in future work.

\section{Stock Market Prediction Methods}

In this section, we will consider the different prediction methods that are available for predicting stock market movements and returns. Some of these methods that will be covered in depth in this section are Technical Analysis, Linear Time Series Models and Machine Learning Models.

\subsection{Technical Analysis}

The idea behind technical analysis is that stock prices move in trends dictated by the constantly changing attitudes of investors in response to different forces. Future stock movements are predicted by using price, volume and observing trends that are dominating the market. Technical analysis rests on the assumption that history repeats itself and that future market direction can be determined by examining past prices [1]. The groups of pro- fessionals who subscribe to this method are the technical analysts or the chartists, as they are more commonly known. To them all information about earnings, dividends and future performance of the company is already reflected in the stock's price history. Therefore the historical price chart is all a chartist needs to make predictions of future stock price movements.

This method of predicting the market is highly criticized because it is highly subjective. Two technical analysts studying the same chart may interpret them differently, thereby arriving at completely different trading strategies. Also a chartist may only occasionally be successful if trends perpetuate. Technical analysis is also considered to be controversial as it contradicts the Efficient Market Hypothesis. Despite such criticism and controversy, the method of technical analysis is used by approximately $90 \%$ of the major stock traders.

In this paper, several technical indicators are used. I will show the details of the technical indicators blow:

1) Moving Average: This indicator returns the moving average of a field over a given period of time. This is done primarily to avoid noise in the daily price movements. The formula of MA used in this chapter is showed below:

$$
M A_{n}=\text { mean (last } \mathrm{n} \text { close prices) }
$$

The $n$ is the parameter. We set $n$ as 10 and 25 in this paper.

2) Oscillator: This function compares a security's closing price to its price range over a given time period. The formula of SO used in this chapter is showed below:

$$
\begin{gathered}
\% K=100 \times \frac{\text { close price }-L_{n}}{H_{n}-L_{n}} \\
\% D=3-\text { period moving average of } \% K
\end{gathered}
$$

where $H_{n}$ and $L_{n}$ are respectively the highest and the lowest price over the last $n$ periods. The $n$ is the parameter. We set $n$ as 10 .

3) Volatility: Volatility can either be measured by using the standard deviation or variance between returns from the stock or market index. Commonly, the higher the volatility, the riskier the stock or market. The formula of volatility used in this chapter is showed below:

$$
\text { Volatility }=\operatorname{std}(\text { last } n \text { close prices })
$$

The $n$ is the parameter. We set $n$ as 10 .

Beside the technical indicators above, we also used some simple technical indicators: return, actual price change, volume and volume difference.

\subsection{Linear Time Series Models}

Linear time series models are often used to predict future values of the time series by detecting linear relationships 
between the historical data of the stock and the time series under consideration. [2] Depending on the number of different variables used as factors of the time series, two different types of linear time series models are used. For the case where only one factor is used to predict the time series, univariate regression is employed. If more variables are used to predict the time series, then the model of multivariate regression is used.

The regression method works by having a set of independent variables, whole linear combination gives the predicted value of the time series under consideration. The predicted value of the time series is thus called the dependant variable. The model associated with such a regression method is given by the Equation (5) below:

$$
y_{t}=\sum_{n=1}^{m} a_{n} x_{n, t}
$$

where $y_{t}$ is the dependent variable of the time series at time $t, a_{n}$ is the regression coefficient and $x_{n, t}$ is the independent variable(s). For univariate regression, $m=1$, whereas for multivariate regression, $m>1$.

In this paper, linear regression model will be used. Regression models are statistical models that are used to predict one variable from one or more other variables. Inference based on such models is called Regression analysis, which is the technique for modeling and analyzing several variables, when the focus is on the relationship between a dependent variable and one or more independent variables. More specifically, the regression model helps in understanding how the typical value of the dependent variable changes when any one of the independent variables is varied.

Given a data set $\left\{y_{i}, x_{i 1}, \ldots, x_{i p}\right\}_{i=1}^{n}$ of n statistical units, a linear regression model assumes that the relationship between the dependent variable $y_{i}$ and the p-vector of regressors $x_{i}$ is approximately linear. This approximate relationship is modeled through a "disturbance term" $\varepsilon_{i}$ - an unobserved random variable that adds noise to the linear relationship between the dependent variable and regressors. The model is described by the function given below:

$$
y_{i}=\beta_{1} x_{i 1}+\cdots+\beta_{p} x_{i p}+\varepsilon_{i}=x_{i}^{\prime} \beta+\varepsilon_{i} \quad i=1, \ldots, n
$$

Here $y_{i}$ is the forecasted return or volatility that is based on $\mathrm{p}$ independent variables, $x_{i 1}$ to $x_{i p}$ and $\beta_{1}$ to $\beta_{p}$ are the coefficients of the linear regression model.

These $\mathrm{n}$ equations are often stacked together and written in vector form as:

$$
y=X \beta+\varepsilon
$$

where

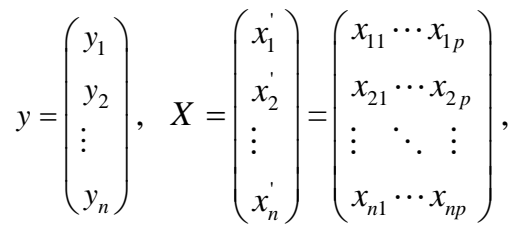

$$
\begin{aligned}
& \beta=\left(\begin{array}{l}
\beta_{1} \\
\beta_{2} \\
\vdots \\
\beta_{p}
\end{array}\right), \varepsilon=\left(\begin{array}{l}
\varepsilon_{1} \\
\varepsilon_{2} \\
\vdots \\
\varepsilon_{p}
\end{array}\right) .
\end{aligned}
$$

The study using the linear regression model is achieved using the "regress" function in MATLAB, which takes in the inputs to the model and the desired output from the model and returns the coefficients of the linear regression model. The coefficients of the linear regression model are obtained by the least mean squares method, which minimizes an error function which is the square of the error of each predicted value.

\subsection{Machine Learning Models}

Machine learning models [3] are a class of models which can study the underlying relationships between the independent variables and the dependent variables of the time series by being "trained" on a sample set of data which should ideally be representative of the actual environment. The most popular machine learning model used for stock market prediction is that of neural networks (NNs), thus my research work will be focusing on the use of NNs in predicting the Shanghai Composite Index returns.

$\mathrm{NN}$ is a powerful data modeling tool that is able to capture and map an input (independent variable) set to a corresponding output (dependent variable) set. The motivation for the development of the NN technology stemmed from the desire to develop an artificial system that could perform "intelligent" tasks similar to those performed by the human brain. A NN can resemble the human brain in two ways:

1) A NN acquires knowledge through learning.

2) A NN's knowledge is stored within inter-neuron connection strengths known as synaptic weights.

The NN architecture can be used to represent both linear and non-linear relationships. For data that contains nonlinear characteristics, traditional linear models are simply inadequate. The most common neural network model is that of the Multi-Layer Perceptron (MLP) and this study on the Chinese stock market prediction will focus on the MLP. The MLP is also known as the supervised network because it requires a desired output in order to learn. The goal of this type of network is to create a model that correctly maps the input to the output using historical data so that the model can then be used to produce the output 
when the desired output is unknown. A graphical representation of an MLP with two hidden layers is shown in Figure 1 below:

The MLP is in fact a distributed processing network, comprising of numerous neurons, with each neuron as the most basic processing element within the network [12]. A neuron is a processing unit that takes in a number of inputs and gives a distinct output for the input it receives. The inputs are fed to each neuron through links between the different layers. An MLP only allows links between successive layers of neurons. Each link is characterized by a weight value, and it is this weight value where the "memory" or knowledge of the problem is stored. The output of each neuron is determined jointly by the weighted sum of the inputs, as well as the activetion function, $f$, used in the neuron. The most commonly used activation functions are the hardlimit, linear, sigmoid and tansigmoid activation functions.

As depicted in Figure 1, the MLP is made up of a number of layers of neurons. The input layer defines the inputs to the MLP. The inputs are then passed on to the first hidden layer of the MLP. For an MLP, the number of hidden layers must be at least one. After propagating through all the hidden layers, the input finally reaches the output layer, which then gives the final output of the whole network for the given set of inputs.

A common notation to represent the architecture of the MLP is to use the string R-S1-S2-S3, where R is the number of inputs to the MLP, S1 and S2 indicates the number of neurons in the first and second hidden layer respectively, and S3 indicates the number of neurons in the output layer, which is also the number of outputs in the output set of the network.

After the architecture of the MLP has been decided, the network will have to be trained before it can be used in any application. This procedure of training involves modifying the weights of the links within the MLP so that the MLP will store the correct knowledge of the system which it is modeling. The training procedure for

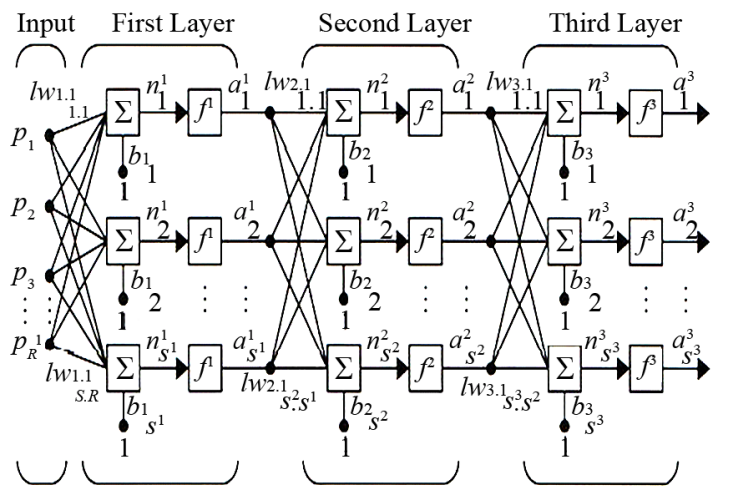

Figure 1. Architecture of MLP with two hidden layers [3]. an MLP can be done using a back-propagation algorithm to update the all the weights of the neurons in order to derive a good 'fit' on the training data, but at the same time not sacrificing performance on the unseen data. This means that a well-trained MLP must be able to generalize well from the training data that is presented to it.

\section{Predictability of Shanghai Composite Index Return}

In this section, we firstly introduce the simulation design which consists of data collection, data pre-processing, three comparison experiments and the metrics for performance evaluation, then the simulation results and discussions are showed.

\subsection{Simulation Design}

\subsubsection{Data Collection}

We collected the historical data of Shanghai Composite Index for both daily data and weekly data from the year 2000 to the year 2010 from the stockstar website [13].

\subsubsection{Data Pre-Processing}

Because we want to see whether the return is random or not, we calculate the daily returns from the daily data, the weekly returns from the weekly data for the Shanghai Composite Index. The entire set is divided into a three separate data sets for different usage. The first data is called the "Training" data set and is used for training and adjusting the coefficients or weights of the systems. The second is the 'Verification' data set which is used for verifying the predictive performance of the trained systems and evaluating the choice of parameters for a good trading system. Finally the third data set or 'Test' data set is used for an actual trading test to determine the trading performance of the chosen trading system. We set the training data from 2000 to 2006, the verification data from 2007 to 2008 and the test data from 2009 to 2010.

\subsubsection{Predictability Experiments of Shanghai Composite Index return}

The study for the predictability of daily and weekly Shanghai Composite Index return is tested using three experiments:

1) In Experiment I, 10 lags of Shanghai Composite Index returns are used for the prediction of the subsequent period's return.

2) In Experiment II, the actual Shanghai Composite Index returns of up to 10 lags, 10-period moving average of closing Shanghai Composite Index values, 25-period moving average of the same and a 10-period oscillator is used for the prediction of the subsequent period's return.

3) In Experiment III, the actual Shanghai Composite Index returns of up to 10 lags, 10-period moving average of closing Shanghai Composite Index values, 25-period 
moving average of the same and a 10-period volatility indicator is used for the prediction of the subsequent period's return.

In Experiments II and III, the term "period” refers to daily or weekly based on the context of the experiment. In each of the three experiments, the efficacy of the regression and neural network models in predicting the subsequent period's Shanghai Composite Index return is evaluated.

\subsubsection{Metrics Used for Performance Evaluation}

The performance of all the trading systems used in this paper will be accessed using two metrics:

\subsubsection{Directional Accuracy}

The first metric is the percentage of correct signs of predicted returns as compared to the actual returns. This is termed as directional accuracy in this paper. It has been argued in literature that for prediction on the stock market, the signs of returns are more important than the actual magnitude of returns. Also, it has been shown by Pesaran and Timmermann [2] that directional accuracy measures has a higher correlation with returns compared to using the mean square error.

\subsubsection{Annual Return Rate}

The second metric is the annual return rate (ARR) from simulated trading. The ARR indicates the annual returns from trading with an initial investment of 1 (ARR of 1.1 indicates a $10 \%$ profit). In this paper, both trading with and without short selling has been considered. Also, both the cases with and without commission costs are discussed to show the effects of commission costs when the trading systems are in actual use. As mentioned earlier, commission costs play a significant role when the number of transactions gets large.

In this paper, the commission cost is assumed to be $0.2 \%$ per trade (a single trade indicates either a buying or selling decision), which is a rather conservative amount. In computing the ARR for trading performance evaluation, the cumulative returns for the whole period (training or verification) is calculated first. After which, the ARR is obtained by taking the nth root of the cumulative returns, where $n$ is the number of years in the period. In calculating the cumulative returns, two possibilities exist depending whether a long or short position is held. In the case of a long position, the cumulative return after period $t$ is calculated as:

$$
\begin{aligned}
& f \text { (Cumulative Returns })_{t}=
\end{aligned}
$$

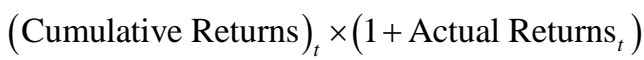

For a short position, the cumulative return in period $\mathrm{t}$ is:

$$
\begin{aligned}
& \text { (Cumulative Returns })_{t}= \\
& \text { (Cumulative Returns })_{t} \times\left(1-\text { Actual Returns }_{t}\right. \text { ) }
\end{aligned}
$$

For the trading decision made in this chapter, the threshold-based trading rule is used. The threshold based trading rule is based on both the magnitude and signs of predictions made by the systems. This decision-making trading rule is used to make trading decisions via the following clauses:

1) If the predicted return rate is positive and its magnitude greater than the threshold value, then a long (buy) position is recommended.

2) Alternatively, if the predicted return rate is negative and its magnitude greater than the threshold, then a short (sell) position is recommended.

3) If the above conditions fail, 3 scenarios are possible whereby the recommendation is to stay away from the market. If already in a long position, withdraw from market if the predicted return rate is negative. On the other hand, if already in a short position, withdraw from market if the return rate is positive. Else, the current position is maintained.

The use of this threshold-based trading rule leads to the need to vary the threshold value used in order to find an appropriate value for the trading system which leads to good trading performances.

\subsection{Simulation Results}

\subsubsection{Predictability Experiments of Shanghai Composite Index Return}

For convenience, in the presentation of tables, we denote directional accuracy as DA, annual return rate as ARR and commission fee as CF.

For daily data, we firstly use the regression model to do the prediction. We show the experiment results in Tables 1-3:

Table 1. Performance of regression model in experiment I (daily).

\begin{tabular}{cccccc}
\hline \multirow{2}{*}{ Period } & \multirow{2}{*}{ DA } & \multicolumn{2}{c}{ ARR (No short sell) } & \multicolumn{2}{c}{ ARR (Short sell) } \\
\cline { 3 - 6 } & & No CF & $0.2 \%$ CF & No CF & $0.2 \%$ CF \\
\hline \multirow{2}{*}{ Training } & $54.21 \%$ & 1.2879 & 1.2760 & 1.2579 & 1.2330 \\
Verification & $54.81 \%$ & 1.0698 & 1.0511 & 1.7173 & 1.6586 \\
\hline
\end{tabular}

Table 2. Performance of regression model in experiment II (daily).

\begin{tabular}{cccccc}
\hline \multirow{2}{*}{ Period } & \multirow{2}{*}{ DA } & \multicolumn{2}{c}{ ARR (No short sell) } & \multicolumn{2}{c}{ ARR (Short sell) } \\
\cline { 3 - 6 } & & No CF & $0.2 \%$ CF & No CF & $0.2 \%$ CF \\
\hline Training & $52.90 \%$ & 1.2154 & 1.1960 & 1.2855 & 1.2462 \\
Verification & $55.65 \%$ & 0.9676 & 0.9620 & 1.4507 & 1.4336 \\
\hline
\end{tabular}


In experiment I, the threshold for trading is 0.0008. In experiment II, the threshold for trading is 0.0002 . In experiment III, the threshold for trading is 0.0006 . From the Tables 1-3, we can see that the experiment I showed the best performance of regression model. So we choose the method in experiment I for test period. We show the result in Table 4.

In experiment I, the threshold for trading is 0.0018 and the number of nodes is 18 . In experiment II, the threshold for trading is 0.0016 and the number of nodes is 18 . In experiment III, the threshold for trading is 0.0014 and the number of nodes is 12 . From the Tables 5-7, we can see that the experiment II showed the best performance of NN model. So we choose the method and parameters in experiment II for test period. We show the result in Table 8.

Table 3. Performance of regression model in experiment III (daily).

\begin{tabular}{cccccc}
\hline \multirow{2}{*}{ Period } & \multirow{2}{*}{ DA } & \multicolumn{2}{c}{ ARR (No short sell) } & \multicolumn{2}{c}{ ARR (Short sell) } \\
\cline { 3 - 6 } & & No CF & $0.2 \%$ CF & No CF & $0.2 \%$ CF \\
\hline Training & $53.68 \%$ & 1.2690 & 1.2581 & 1.3955 & 1.3714 \\
Verification & $57.11 \%$ & 0.9434 & 0.9380 & 1.1235 & 1.1116 \\
\hline
\end{tabular}

Table 4. Performance of regression model in experiment I (daily).

\begin{tabular}{cccccc}
\hline \multirow{2}{*}{ Period } & \multirow{2}{*}{ DA } & \multicolumn{2}{c}{ ARR (No short sell) } & \multicolumn{2}{c}{ ARR (Short sell) } \\
\cline { 3 - 6 } & & No CF & $0.2 \%$ CF & No CF & $0.2 \%$ CF \\
\hline \multirow{2}{*}{ Testing } & $56.63 \%$ & 1.2638 & 1.2572 & 1.3351 & 1.3299 \\
\hline
\end{tabular}

Table 5. Performance of NN model in experiment I (daily).

\begin{tabular}{cccccc}
\hline \multirow{2}{*}{ Period } & \multirow{2}{*}{ DA } & \multicolumn{2}{c}{ ARR (No short sell) } & \multicolumn{2}{c}{ ARR (Short sell) } \\
\cline { 3 - 6 } & & No CF & $0.2 \%$ CF & No CF & $0.2 \%$ CF \\
\hline Training & $55.89 \%$ & 1.4654 & 1.4458 & 1.8384 & 1.7981 \\
Verification & $55.86 \%$ & 1.1324 & 1.1118 & 1.7182 & 1.6624 \\
\hline
\end{tabular}

Table 6. Performance of NN model in experiment II (daily).

\begin{tabular}{cccccc}
\hline \multirow{2}{*}{ Period } & \multirow{2}{*}{ DA } & \multicolumn{2}{c}{ ARR (No short sell) } & \multicolumn{2}{c}{ ARR (Short sell) } \\
\cline { 3 - 6 } & & No CF & $0.2 \%$ CF & No CF & $0.2 \%$ CF \\
\hline Training & $58.88 \%$ & 1.7051 & 1.6812 & 2.1571 & 2.1003 \\
Verification & $55.60 \%$ & 1.0135 & 1.0032 & 1.2261 & 1.2026 \\
\hline
\end{tabular}

Table 7. Performance of NN model in experiment III (daily).

\begin{tabular}{cccccc}
\hline \multirow{2}{*}{ Period } & \multirow{2}{*}{ DA } & \multicolumn{2}{c}{ ARR (No short sell) } & \multicolumn{2}{c}{ ARR (Short sell) } \\
\cline { 3 - 6 } & & No CF & $0.2 \%$ CF & No CF & $0.2 \%$ CF \\
\hline Training & $55.37 \%$ & 1.3453 & 1.3325 & 1.4782 & 1.4493 \\
Verification & $55.81 \%$ & 0.9581 & 0.9527 & 1.2554 & 1.2419 \\
\hline
\end{tabular}

For the weekly data, we firstly use the regression model to do the prediction. We show the experiment results in Tables 9-11.

In experiment I, the threshold for trading is 0.0014 . In experiment II, the threshold for trading is 0.0008 . In experiment III, the threshold for trading is 0.0002 . From the Tables 9-11 above, we can see that the experiment II showed the best performance of regression model. So we choose the method in experiment III for test period. We show the result in Table 12 below.

Table 8. Performance of NN model in experiment II (daily).

\begin{tabular}{cccccc}
\hline \multirow{2}{*}{ Period } & \multirow{2}{*}{ DA } & \multicolumn{2}{c}{ ARR (No short sell) } & \multicolumn{2}{c}{ ARR (Short sell) } \\
\cline { 3 - 6 } & & No CF & $0.2 \%$ CF & No CF & $0.2 \%$ CF \\
\hline \multirow{2}{*}{ Testing } & $56.02 \%$ & 1.2984 & 1.2569 & 1.3966 & 1.3517 \\
\hline
\end{tabular}

Table 9. Performance of regression model in experiment $\mathbf{I}$. (weekly)

\begin{tabular}{cccccc}
\hline \multirow{2}{*}{ Period } & \multirow{2}{*}{ DA } & \multicolumn{2}{c}{ ARR (No short sell) } & \multicolumn{2}{c}{ ARR (Short sell) } \\
\cline { 3 - 6 } & & No CF & $0.2 \%$ CF & No CF & $0.2 \%$ CF \\
\hline Training & $62.86 \%$ & 1.2441 & 1.2416 & 1.3613 & 1.3563 \\
Verification & $56.67 \%$ & 1.0308 & 1.0287 & 1.4773 & 1.4711 \\
\hline
\end{tabular}

Table 10. Performance of regression model in experiment II (weekly).

\begin{tabular}{cccccc}
\hline \multirow{2}{*}{ Period } & DA & \multicolumn{2}{c}{ ARR (No short sell) } & \multicolumn{2}{c}{ ARR (Short sell) } \\
\cline { 3 - 6 } & & No CF & $0.2 \%$ CF & No CF & $0.2 \%$ CF \\
\hline Training & $59.40 \%$ & 1.2398 & 1.2356 & 1.3735 & 1.3646 \\
Verification & $66.67 \%$ & 1.1800 & 1.1781 & 1.7099 & 1.7048 \\
\hline
\end{tabular}

Table 11. Performance of regression model in experiment III (weekly).

\begin{tabular}{cccccc}
\hline \multirow{2}{*}{ Period } & DA & \multicolumn{2}{c}{ ARR (No short sell) } & \multicolumn{2}{c}{ ARR (Short sell) } \\
\cline { 3 - 6 } & & No CF & $0.2 \%$ CF & No CF & $0.2 \%$ CF \\
\hline \multirow{2}{*}{ Training } & $59.06 \%$ & 1.1035 & 1.0980 & 1.1013 & 1.0902 \\
Verification & $44.57 \%$ & 0.8628 & 0.8590 & 0.9303 & 0.9221 \\
\hline
\end{tabular}

Table 12. Performance of regression model in experiment II (weekly).

\begin{tabular}{cccccc}
\hline & & \multicolumn{2}{c}{ ARR (No short sell) } & \multicolumn{2}{c}{ ARR (Short sell) } \\
\cline { 3 - 6 } Period & DA & No CF & $0.2 \%$ CF & No CF & $0.2 \%$ CF \\
& & & 0.9039 & 0.9020 & 0.8988 \\
\hline
\end{tabular}


For the weekly data, we then use the NN model to do the prediction. We show the experiment results in Tables 13-15.

In experiment I, the threshold for trading is 0.0010 and the number of nodes is 14 . In experiment II, the threshold for trading is 0.0020 and the number of nodes is 12 . In experiment III, the threshold for trading is 0.0014 and the number of nodes is 20 . From the Table $13 \sim 15$, we can see that the experiment II showed the best performance of NN model. So we choose the method and parameters in experiment II for test period. We show the result in Table 16.

From the results showed above, we can see that the performance of NN model is better than the performance of regression model. We also can find that for the daily data, the ARRs of both regression model and NN model are better than the ARRs of buy-and-hold strategy in testing period (testing period ARR is 1.2419). Unfortunately, for the weekly data, the ARRs of both regression model and NN model are worse than the ARRs of buyand-hold strategy.

Table 13. Performance of NN model in experiment I (weekly).

\begin{tabular}{cccccc}
\hline \multirow{2}{*}{ Period } & DA & \multicolumn{2}{c}{ ARR (No short sell) } & \multicolumn{2}{c}{ ARR (Short sell) } \\
\cline { 3 - 6 } & & No CF & $0.2 \%$ CF & No CF & $0.2 \%$ CF \\
\hline Training & $66.29 \%$ & 1.4186 & 1.4122 & 1.6872 & 1.6728 \\
Verification & $53.33 \%$ & 1.0847 & 1.0795 & 1.5257 & 1.5108 \\
\hline
\end{tabular}

Table 14. Performance of NN model in experiment II (weekly).

\begin{tabular}{cccccc}
\hline \multirow{2}{*}{ Period } & \multirow{2}{*}{ DA } & \multicolumn{2}{c}{ ARR (No short sell) } & \multicolumn{2}{c}{ ARR (Short sell) } \\
\cline { 3 - 6 } & & No CF & $0.2 \%$ CF & No CF & $0.2 \%$ CF \\
\hline Training & $62.39 \%$ & 1.3135 & 1.3081 & 1.4347 & 1.4242 \\
Verification & $56.67 \%$ & 1.0496 & 1.0480 & 1.3380 & 1.3337 \\
\hline
\end{tabular}

Table 15. Performance of NN model in experiment III (weekly).

\begin{tabular}{cccccc}
\hline \multirow{2}{*}{ Period } & \multirow{2}{*}{ DA } & \multicolumn{2}{c}{ ARR (No short sell) } & \multicolumn{2}{c}{ ARR (Short sell) } \\
\cline { 3 - 6 } & & No CF & $0.2 \%$ CF & No CF & $0.2 \%$ CF \\
\hline Training & $65.63 \%$ & 1.1271 & 1.1237 & 1.1744 & 1.1673 \\
Verification & $52.17 \%$ & 1.0074 & 1.0039 & 1.1390 & 1.1313 \\
\hline
\end{tabular}

Table 16. Performance of NN model in experiment II (weekly).

\begin{tabular}{cccccc}
\hline \multirow{2}{*}{ Period } & \multirow{2}{*}{ DA } & \multicolumn{2}{c}{ ARR (No short sell) } & \multicolumn{2}{c}{ ARR (Short sell) } \\
\cline { 3 - 6 } & & No CF & $0.2 \%$ CF & No CF & $0.2 \%$ CF \\
\hline Testing & $62.77 \%$ & 1.0613 & 1.0598 & 1.2287 & 1.2258 \\
\hline
\end{tabular}

\section{Predictability of Shanghai Composite Index Price Volatility}

In this section, we firstly introduce the simulation design which consists of data collection, data pre-processing, three comparison experiments and the metrics for performance evaluation, then the simulation results and discussions are showed.

\subsection{Simulation Design}

\subsubsection{Data Collection}

We collected the historical data of Shanghai Composite Index for both daily data and weekly data from the year 2000 to the year 2010 from the stockstar website [13].

\subsubsection{Data Pre-Processing}

Because we want to see whether the return is random or not, we calculate the daily returns from the daily data, the weekly returns from the weekly data for the Shanghai Composite Index. The entire set is divided into a three separate data sets for different usage. The first data is called the "Training" data set and is used for training and adjusting the coefficients or weights of the systems. The second is the "Verification" data set which is used for verifying the predictive performance of the trained systems and evaluating the choice of parameters for a good trading system. Finally the third data set or "Test" data set is used for an actual trading test to determine the trading performance of the chosen trading system. We set the training data from 2000 to 2006, the verification data from 2007 to 2008 and the test data from 2009 to 2010.

\subsubsection{Predictability Experiments of Shanghai Composite Index Price Volatility}

The study for the predictability of daily and weekly Shanghai Composite Index price-changes is tested using three experiments:

1) In Experiment IV, 10 lags of actual Shanghai Composite Index closing price values and 10 lags of periodic price-changes are used for the prediction of the subsequent period's price-change.

2) In Experiment V, 10 lags of actual Shanghai Composite Index trading volume values AND 10 lags of periodic trading volume differences are used for the prediction of the subsequent period's price-change.

3) In Experiment VI, 10 lags of actual Shanghai Composite Index closing price values, 10 lags of periodic price-changes, 10 lags of trading volume values and 10 lags of periodic trading volume differences are used for the prediction of the subsequent period's price change.

\subsubsection{Metrics Used for Performance Evaluation}

The performance of all the trading systems used in this paper will be accessed only using one metric: 
Directional Accuracy

This metric is the percentage of correct signs of predicted returns as compared to the actual returns. This is termed as directional accuracy in this chapter. It has been argued in literature that for prediction on the stock market, the signs of returns are more important than the actual magnitude of returns. Also, it has been shown by Pesaran and Timmermann [2] that directional accuracy measures has a higher correlation with returns compared to using the mean square error.

\subsection{Simulation Results}

\subsubsection{Predictability Experiments of Shanghai Composite Index Price Volatility}

For daily data, we firstly use the regression model to do the prediction. We show the experiment results in Tables 17-19.

From the Tables 17-19, we can see that the experiment VI showed the best performance of regression model. So we choose the method in experiment VI for test period. We show the result in Table 20.

For the daily data, we then use the NN model to do the prediction. We show the experiment results in Tables 21-23.

Table 17. Performance of regression model in experiment IV (daily).

\begin{tabular}{cc}
\hline Period & Directional Accuracy \\
\hline Training & $52.96 \%$ \\
Verification & $56.28 \%$ \\
\hline
\end{tabular}

Table 18. Performance of regression model in experiment $\mathrm{V}$ (daily).

\begin{tabular}{cc}
\hline Period & Directional Accuracy \\
\hline Training & $54.27 \%$ \\
Verification & $54.39 \%$ \\
\hline
\end{tabular}

Table 19. Performance of regression model in experiment VI (daily).

\begin{tabular}{cc}
\hline Period & Directional Accuracy \\
\hline Training & $56.84 \%$ \\
Verification & $55.02 \%$ \\
\hline
\end{tabular}

Table 20. Performance of regression model in experiment VI (daily).

\begin{tabular}{cc}
\hline Period & Directional Accuracy \\
\hline Testing & $57.36 \%$ \\
\hline
\end{tabular}

In experiment IV, the number of nodes is 16 . In experiment $\mathrm{V}$, the number of nodes is 10 . In experiment III, the number of nodes is 10. From the Tables 21-23, we can see that the experiment VI showed the best performance of NN model. So we choose the method and parameters in experiment VI for test period. We show the result in Table 24.

For the weekly data, we firstly use the regression model to do the prediction. We show the experiment results in Tables 25-27.

Table 21. Performance of NN model in experiment IV (daily).

\begin{tabular}{cc}
\hline Period & Directional Accuracy \\
\hline Training & $55.98 \%$ \\
Verification & $54.23 \%$ \\
\hline
\end{tabular}

Table 22. Performance of NN model in experiment V (daily).

\begin{tabular}{cc}
\hline Period & Directional Accuracy \\
\hline Training & $53.14 \%$ \\
Verification & $53.49 \%$ \\
\hline
\end{tabular}

Table 23. Performance of NN model in experiment VI (daily).

\begin{tabular}{cc}
\hline Period & Directional Accuracy \\
\hline Training & $57.29 \%$ \\
Verification & $55.60 \%$ \\
\hline
\end{tabular}

Table 24. Performance of NN model in experiment VI (daily).

\begin{tabular}{cc}
\hline Period & Directional Accuracy \\
\hline Testing & $58.18 \%$ \\
\hline
\end{tabular}

Table 25. Performance of regression model in experiment IV (weekly).

\begin{tabular}{cc}
\hline Period & Directional Accuracy \\
\hline Training & $59.70 \%$ \\
Verification & $54.39 \%$ \\
\hline
\end{tabular}

Table 26. Performance of regression model in experiment $\mathrm{V}$ (weekly).

\begin{tabular}{cc}
\hline Period & Directional Accuracy \\
\hline Training & $59.70 \%$ \\
Verification & $54.35 \%$ \\
\hline
\end{tabular}

Table 27. Performance of regression model in experiment VI (weekly).

\begin{tabular}{cc}
\hline Period & Directional Accuracy \\
\hline Training & $61.10 \%$ \\
Verification & $56.74 \%$ \\
\hline
\end{tabular}


From the Tables 25-27, we can see that the experiment VI showed the best performance of regression model. So we choose the method in experiment VI and the parameters for test period. We show the result in Table $\mathbf{2 8}$.

For the weekly data, we then use the NN model to do the prediction. We show the experiment results in Tables 29-31.

In experiment $\mathrm{I}$, the number of nodes is 12 . In experiment II, the number of nodes is 20. In experiment III, the number of nodes is 16. From the Tables 29-31, we can see that the experiment VI showed the best performance of NN model. So we choose the method and parameters in experiment VI for test period. We show the result in Table 32.

Similar with the conclusions of the experiment I, II and III, from the results showed above, we can see that the performance of NN model is better than the performance of regression model.

Table 28. Performance of regression model in experiment VI (weekly).

\begin{tabular}{cc}
\hline Period & Directional Accuracy \\
\hline Testing & $59.66 \%$ \\
\hline
\end{tabular}

Table 29. Performance of NN model in experiment IV (weekly).

\begin{tabular}{cc}
\hline Period & Directional Accuracy \\
\hline Training & $61.19 \%$ \\
Verification & $58.70 \%$ \\
\hline
\end{tabular}

Table 30. Performance of NN model in experiment V (weekly).

\begin{tabular}{cc}
\hline Period & Directional Accuracy \\
\hline Training & $61.97 \%$ \\
Verification & $56.70 \%$ \\
\hline
\end{tabular}

Table 31. Performance of NN model in experiment VI (weekly).

\begin{tabular}{cc}
\hline Period & Directional Accuracy \\
\hline Training & $65.79 \%$ \\
Verification & $58.52 \%$ \\
\hline
\end{tabular}

Table 32. Performance of NN model in experiment VI (weekly).

\begin{tabular}{cc}
\hline Period & Directional Accuracy \\
\hline Testing & $59.70 \%$ \\
\hline
\end{tabular}

\section{Conclusions and Future Work}

In this paper, we do the prediction of Shanghai Composite Index return and the prediction of Shanghai Composite Index volatility based on regression model and NN model using the daily and weekly data of Shanghai Composite Index. The directional accuracy of most of the experiments is beyond $55 \%$. For the prediction of Shanghai Composite Index return, both trading with and without short selling has been considered, and the results show in most cases, trading with short selling leads to higher profits. Also, both the cases with and without commission costs are discussed to show the effects of commission costs when the trading systems are in actual use. We find that the performance of NN model is better than the performance of regression model. We also find that for the daily data, the ARRs of both regression model and NN model are better than the ARRs of buy-and-hold strategy in testing period (testing period ARR is 1.2419). Unfortunately, for the weekly data, the ARRs of both regression model and NN model are worse than the ARRs of buy-and-hold strategy in testing period. For the predicttion of Shanghai Composite Index volatility, we can find similar conclusion that the performance of $\mathrm{NN}$ model is better than the performance of regression model.

For the future work, two aspects may be considered. The first aspect: it has been studied in literature that better performance can be achieved by using systems comprising of multiple models. For example, three or four models could be used within each system, and a trend classification algorithm can be use to classify the time series into a larger number of different trends. The second aspect: the input data used for predictions of markets can be extended by using macro-fundamental data such as interest rate and required reserve ratio. Such macrofundamental data may contain useful information which can be used to predict market movements more accurately.

\section{REFERENCES}

[1] B. G. Malkiel, “A Random Walk Down Wall Street,” W. W. Norton \& Company, New York and London, 1999.

[2] M. H. Pesaran and A. Timmermann, "Forecasting Stock Returns: An Examination of Stock Market Trading in the Presence of Transaction Costs," Journal of Forecasting, Vol. 13, No. 4, 1994, pp. 335-367. doi:10.1002/for.3980130402

[3] M. T. Mitchell, "Machine Learning,” The McGraw-Hill Companies, New York, 1997.

[4] M. H. Eng and Q.-G. Wang, "Modeling of Stock Markets with Mean Reversion,” The 6th IEEE International Conference on Control and Automation (IEEE ICCA 2007), Guangzhou, 30 May-1 June 2007, pp. 2615-2618.

[5] J.-H. Wang and J.-Y. Leu, "Stock Market Trend Predic- 
tion Using ARIMA-Based Neural Networks,” The 1996 IEEE International Conference on Neural Networks, Washington DC, 3-6 June 1996, pp. 2160-2165.

[6] W. Wang, D. Okunbor and F. C. Lin, "Future Trend of the Shanghai Stock Market," ICONIP '02: Proceedings of the 9th International Conference on Neural Information Processing, Singapore, 18-22 November, pp. 2320-2324.

[7] L.-X. Liu and J.-H Ma, "Multivariate Nonlinear Prediction of Shenzhen Stock Price," The 3rd International Conference on Wireless Communications, Networking and Mobile Computing (WiCOM 2007), Shanghai, 21-23 September 2007, pp. 4120-4123.

[8] S.-H. Chen, C.-Q Tao and W. He, "A New Algorithm of Neural Network and Prediction in China Stock Market," Pacific-Asia Conference on Circuits, Communications and Systems, PACCS 2009, Chengdu, 16-17 May 2009, pp. 686-689.

[9] X. Xiong, X,-T, Zhang, W, Zhang and C,-Y, Li, "Wave-
let-Based Beta Estimation of China Stock Market,” Proceedings of 2005 International Conference on Machine Learning and Cybernetics, Guangzhou, 18-21 August 2005, pp. 3501-3505.

[10] W.-R. Pan, "Empirical Analysis of Stock Returns Volatility in China Market Based on Shanghai and Shenzhen 300 Index,” 2010 International Conference on Financial Theory and Engineering (ICFTE), Dubai, 18-20 June 2010, pp. 17-21.

[11] X.-M. Song and H.-X. Pan, "Analysis of China Stock Market: Volatility and Influencing Factors,” 2010 International Conference on Management and Service Science (MASS), Wuhan, 24-26 August 2010, pp. 1-5. doi:10.1109/ICMSS.2010.5578224

[12] S. Haykin, "Neural Networks: A Comprehensive Foundation”, Prentice-Hall, Saddle River, 1999.

[13] Http://www.stockstar.com/ 